\title{
A New Look at the Visual Performance of Nonparametric Hazard Rate Estimators ${ }^{1}$
}

\author{
Olaf Gefeller ${ }^{\mathrm{a}}$ and Nils Lid Hjort ${ }^{\mathrm{b}}$ \\ ${ }^{a}$ Department of Medical Statistics, University of Göttingen, \\ Humboldtallee 32, D-37073 Göttingen, Germany \\ ${ }^{\mathrm{b}}$ Department of Mathematics and Statistics, University of Oslo, \\ P.B. 1053 Blindern, N-0316 Oslo, Norway
}

\begin{abstract}
Nonparametric curve estimation by kernel methods has attracted widespread interest in theoretical and applied statistics. One area of conflict between theory and application relates to the evaluation of the performance of the estimators. Recently, Marron and Tsybakov (1995) proposed visual error criteria for addressing this issue of controversy in density estimation. Their core idea consists in using integrated alternatives to the Hausdorff distance for measuring the closeness of two sets based on the Euclidean distance. In this paper we transfer these ideas to hazard rate estimation from censored data. We are able to derive similar results that help to understand when the application of the new criteria will lead to answers that differ from those given by the conventional approach.
\end{abstract}

\section{Introduction}

In various areas of applied and theoretical statistics nonparametric curve estimation by kernel methods has attracted widespread interest during the last two decades. Without imposing any distributional assumptions on the observed data, structural information, for example, on the underlying density functions, some interesting functionals of the density (like the hazard rate) or regression curves, can be obtained by "smoothing" the empirical mass of the observations to some neighbouring environment around the observed data points. Starting with the pioneering work by Rosenblatt (1956) and Parzen (1962), nowadays a vast literature on the properties of kernel methods can be found (for recent textbooks see Wand and Jones (1995), Härdle (1991)). Important progress has been made recently in a variety of issues (for example, bandwidth selection, boundary behaviour, software implementation), mostly originating from research in density estimation, the simplest situation. However, it has been criticised repeatedly that there is an obvious gap between theory and application with respect to the evaluation of the performance of the nonparametric estimators. The estimated

${ }^{1}$ A slightly modified version of this paper is to be published in I. Balderjahn, R. Mathar, M. Schader (eds.): Data Highways and Information Flooding, a Challenge for Classification and Data Analysis. Spinger Series "Studies in Classification, Data Analysis, and Knowledge Organization", Volume 8. Springer-Verlag, Berlin, to appear in 1997. 
curves that are judged to be "good" from theoretical reasoning (based on their distance to the true curve in terms of the classical norms on function spaces, mostly $L_{2}$ ) do not satisfy applied statisticians as their "graphical fit" can be substantially poorer than that of other estimators which are more appealing from a graphical notion but exhibit larger deviations from the true curve in $L_{2}$-norm. To address this issue of conflict, Marron and Tsybakov (1995) proposed new visual error criteria for assessing the performance of nonparametric density estimators. The core idea of this concept consists in using integrated alternatives to the Hausdorff distance for measuring the closeness of two sets of points based on the usual Euclidean distance.

The aim of this paper is twofold: first, we review this interesting approach and describe the basic properties of the visual error criteria in Sections 2 and 3. Second, in the following Sections 4 and 5 of this paper, we apply the criteria to nonparametric hazard rate estimation from censored data via kernel estimators. The evolving area of hazard rate estimation is a promising field for these new ideas, because, due to the applicationorientated interpretation of hazard rates in practical survival analysis, qualitative aspects of smoothing performance should be preferred to the exclusive consideration of $L_{2}$-fit. As a first step in this direction, we derive similar results as in the density context showing that a suitable asymptotic bridge can be built between expected visual error criteria and conventional integrated mean squared errors (MISE), incorporating some weight function depending on the derivative of the hazard rate. In the final section we discuss advantages and disadvantages of this approach and point to future work that needs to performed to broaden the understanding of the concept.

\section{Conventional L-norm criteria}

The conventional approach for measuring the distance between some "true" curve $h$ and its estimate $\widehat{h}$ relies on the application of classical mathematical norms on function spaces. The most popular representative of this approach is the $L_{2}$-norm. The distance between two functions in terms of the $L_{2}$-norm is then defined as

$$
L_{2}(\widehat{h}, h)=\int[\widehat{h}(x)-h(x)]^{2} \mathrm{~d} x .
$$

Alternatively, other variants of the broad class of $L$-norms such as, for example, $L_{1}(\widehat{h}, h)=\int|\widehat{h}(x)-h(x)| \mathrm{d} x$ and $L_{\infty}(\widehat{h}, h)=\sup _{x}|\widehat{h}(x)-h(x)|$ have also been proposed, but are less often used. All measurements of distance based on the various $L$-norms have one fundamental property in common: they focus completely on the vertical discrepancy between the curves at all points $x$ belonging to the support of the functions (typically in cases of lifetime data, the support is some subset of $\mathcal{R}_{+}$).

A striking graphical example that the concentration on vertical discrepancy can contradict the visual notion of distance between curves has been 
provided by Kooperberg and Stone (1991). Their example shows a "true" bimodal curve that is approximated by two very different estimates. The key element of this example is that the visually more appealing estimate that recovers the bimodal structure but with some imprecision in the location of the second peak is clearly inferior in terms of all $L$-norms to the other estimate that only recovers the first peak and "smooths away" the lower second peak. The reason for this contradiction between graphical impression and mathematical $L$-norm analysis is obvious: as the distances between the curves are only assessed vertically, no $L$-norm criterion realises that the first estimate is "structurally correct", although quite incorrect with respect to localising the second peak, and thus penalises the estimate in both regions of vertical discrepancy, the region of the true second peak and that of the estimated second peak, whereas the "oversmoothed" estimate is penalised only once, in the region of the true peak. Any remedy to this problem has to drop the strict reliance on vertical distances and has to offer another way of conceptualising discrepancies between curves.

\section{Visual error criteria}

One such alternative approach of measuring the discrepancy between curves has been developed by Marron and Tsybakov (1995) who termed their measures visual error criteria. The starting point for the derivation of these criteria consists in treating curves not as pointwise collections of the values of functions $\widehat{h}$ and $h$ of a single variable at fixed $x \in \mathcal{R}_{+}$but instead as sets in $\mathcal{R}^{2}$ that are defined by their corresponding "graphs". The graph of some function $h$ is defined as the set $G_{h}=\left\{(x, y): x \in \mathcal{R}_{+}, y=h(x)\right\} \subset \mathcal{R}^{2}$. Some planar distance between the sets $G_{h}$ and $G_{\widehat{h}}$ can now replace the conventional vertical distance between $h$ and $\widehat{h}$ for fixed $x \in \mathcal{R}_{+}$. The basis of the definition of a planar distance is the notion of distance from a point to a set, defined as

$$
d\left((x, y), G_{h}\right)=\inf _{\left(x^{*}, y^{*}\right) \in G_{h}}\left\|(x, y)-\left(x^{*}, y^{*}\right)\right\|_{2}
$$

and giving the shortest distance from some fixed point $(x, y)$ to any element in the set $G_{h}$, where $\|\cdot\|_{2}$ denotes the usual Euclidean distance. By allowing $(x, y)$ to vary and take on all elements in the set $G_{\widehat{h}}$, a set of distances between between $G_{h}$ and $G_{\widehat{h}}$ is defined, formally introduced as $\mathcal{M}\left(G_{h}, G_{\widehat{h}}\right)=\left\{d\left((x, y), G_{\widehat{h}}\right):(x, y) \in G_{h}\right\}$. A variety of different ways of summarising the complete information in $\mathcal{M}\left(G_{h}, G_{\widehat{h}}\right)$ when defining a realvalued one-dimensional distance are now conceivable. In analogy with the $L_{2}$-norm criterion based on quadratic vertical distances we first consider the two versions of the quadratic visual error criteria:

$$
\mathrm{VE}_{2}(h \rightarrow \widehat{h})=\left[\int d\left((x, h(x)), G_{\widehat{h}}\right)^{2} \mathrm{~d} x\right]^{1 / 2}
$$




$$
\mathrm{VE}_{2}(\widehat{h} \rightarrow h)=\left[\int d\left((x, \widehat{h}(x)), G_{h}\right)^{2} \mathrm{~d} x\right]^{1 / 2}
$$

As the planar distances are asymmetrical in nature, both versions have to be distinguished since they are not identical in general. However, a symmetrised version of $\mathrm{VE}_{2}$ can also be defined by simply averaging the two versions, in a Pythagorean way, as follows:

$$
\mathrm{SE}_{2}(\widehat{h}, h)=\left[\mathrm{VE}_{2}(h \rightarrow \widehat{h})^{2}+\mathrm{VE}_{2}(\widehat{h} \rightarrow h)^{2}\right]^{1 / 2} .
$$

As for the $L$-norm criteria, similar asymmetrical and symmetrical visual error criteria can also be defined for the non-quadratic distances (by simply integrating over $d\left((x, h(x)), G_{\widehat{h}}\right)$ and $d\left((x, \widehat{h}(x)), G_{h}\right)$, respectively, yielding $\mathrm{VE}_{1}(h \rightarrow \widehat{h})$ and $\mathrm{VE}_{1}(\widehat{h} \rightarrow h)$, respectively) and maximal distance (by considering $\sup \left(\mathcal{M}\left(G_{h}, G_{\widehat{h}}\right)\right)$ and $\sup \left(\mathcal{M}\left(G_{\widehat{h}}, G_{h}\right)\right)$, respectively, yielding $\mathrm{VE}_{\infty}(h \rightarrow \widehat{h})$ and $\mathrm{VE}_{\infty}(\widehat{h} \rightarrow h)$, respectively). The symmetrical version of the two variants of $\mathrm{VE}_{\infty}$ given by

$$
\mathrm{SE}_{\infty}(\widehat{h}, h)=\max \left\{\mathrm{VE}_{\infty}(h \rightarrow \widehat{h}), \mathrm{VE}_{\infty}(\widehat{h} \rightarrow h)\right\}
$$

is also traditionally known as the Hausdorff distance. Thus, all other visual error criteria described in this section can be viewed as integrated alternatives to the Hausdorff distance.

It has to be kept in mind that the other symmetrised versions $\left(\mathrm{SE}_{2}\right.$ and $\left.\mathrm{SE}_{1}\right)$ are - in a mathematical sense - not "distances" on the corresponding function space as they do not satisfy the triangle inequality (see Marron and Tsybakov (1995) for an illustrative counterexample). For the practical application of these criteria, lacking this mathematical property does not seem to be a serious drawback.

\section{Kernel estimation of the hazard rate}

The evolving area of hazard rate estimation from censored data comprises a promising field for the application of the visual error criteria as qualitative aspects of smoothing performance are of primary interest here. In this section the necessary background about the statistical setting and some well-known asymptotic results concerning kernel estimators of the hazard rate are briefly summarised. More detailed reviews on this subject can be found in, for example, Gefeller and Michels (1992), Andersen, Borgan, Gill and Keiding (1993), and Hjort (1996).

Suppose $T_{1}, \ldots, T_{n}$ refer to i.i.d. nonnegative failure times with distribution function $F$ and density function $f$, and $C_{1}, \ldots, C_{n}$ denote i.i.d. nonnegative censoring times with distribution function $G$ and density function $g$. Assume further that failure times $T_{i}$ and censoring times $C_{i}$ are independent for all $i=1, \ldots, n$. Under this setting of the so-called random censorship 
model, which is the simplest and most important special case of models for censored failure time data in the framework of counting process models, the observed data consist of the bivariate sample $\left(X_{1}, \delta_{1}\right), \ldots,\left(X_{n}, \delta_{n}\right)$, where $X_{i}=\min \left(T_{i}, C_{i}\right)$ and $\delta_{i}=I\left\{T_{i} \leq C_{i}\right\}$ for $i=1, \ldots, n$. The censoring indicator $\delta_{i}$ provides information on whether the observed $X_{i}$ refers to a true failure time $\left(\delta_{i}=1\right)$ or to a censoring time $\left(\delta_{i}=0\right)$.

The hazard rate, defined as $h(x)=\lim _{\varepsilon \rightarrow 0}(1 / \varepsilon) P\left(x \leq T_{i}<x+\varepsilon \mid T_{i} \geq\right.$ $x$ ) for $x \geq 0$, has the application-orientated flavour that it can be nicely interpreted as the instantaneous risk of observing the failure event of interest at time $x$. In a variety of applications ranging from survival analysis in a medical context to reliability testing in industrial settings the hazard rate is thus used extensively as a methodological tool to describe variations in risk over time. In these applications qualitative aspects of the structure of the hazard rate are more interesting than the precise location and height of peaks of the function.

The most prominent nonparametric approach to estimate the hazard rate is represented by the kernel estimator with a fixed bandwidth $b$ which is given by

$$
\widehat{h}(x)=\sum_{i=1}^{n} \frac{\delta_{(i)}}{n-i+1} \frac{1}{b} K\left(\frac{x-X_{(i)}}{b}\right),
$$

where $\delta_{(i)}$ refers to the censoring indicator corresponding to the $i$-th element of the order statistic of the observed failure times, $K(\cdot)$ denotes the kernel function (satisfying standard conditions, see below), and the bandwidth parameter $b$ has to be positive. Several variations of this kernel estimator have been suggested allowing the bandwidth to vary with $x$, for example, the nearest neighbour kernel estimator (Gefeller and Dette, 1992), the localbandwidth kernel estimator (Müller and Wang, 1994) or the variable kernel estimator (Schäfer, 1985). For the rest of this paper attention is focussed on the simplest case of the fixed-bandwidth kernel estimator. Modifications for the local-bandwidth and the nearest neighbour kernel estimator are straightforward; however, the variable kernel estimator poses the additional complexity that expressions for the asymptotic MISE have not yet been fully derived and thus conditions under which the results of following section might be transferred to the variable kernel estimator are not yet explicitly available.

A variety of results on the asymptotic behaviour of $\widehat{h}$ ranging from different proofs of consistency to sophisticated elaborations on the optimal order of convergence can be found in the literature. For the purpose of this paper it is sufficient to restrict the attention to a result on the asymptotic MISE which can be easily decomposed into integrals over a squared bias $\left(\mu^{2}(\cdot)\right)$ and a variance part $\left(\sigma^{2}(\cdot)\right)$. The necessary technical assumptions for the asymptotics to work can be stated as follows: 
(K) The kernel function $K(\cdot)$ has to be defined on a compact support $[a, b] \subset \mathcal{R}$ as a bounded symmetrical probability density function having a second derivative that is Lipschitz continuous on $[a, b]$.

(H) The hazard rate $h$ has to be twice continuously differentiable and square integrable on $\mathcal{R}_{+}$.

(B) The sequence of bandwidths $b_{n}$ has to approach zero at a rate slower than $n^{-1}$. The convergence rate in this situation is optimised for the bandwidth sequence $b_{n}=C_{0} n^{-1 / 5}$, with $C_{0}>0$ denoting a special constant.

Given that (K), (H) and (B) hold it has been shown that for $n \rightarrow \infty$ the MISE can be represented as follows (omitting the asymptotically vanishing terms):

$$
\begin{aligned}
\operatorname{MISE}(\widehat{h}, h) & =\int \mathrm{E}[\widehat{h}(x)-h(x)]^{2} \mathrm{~d} x \\
& =\int\left(\left[\frac{1}{2} k_{2}(K) b_{n}^{2} h^{\prime \prime}(x)\right]^{2}+\frac{R(K)}{n b_{n}} \frac{h(x)}{(1-F(x))(1-G(x))}\right) \mathrm{d} x \\
& =\int\left(\mu^{2}(x)+\sigma^{2}(x)\right) \mathrm{d} x,
\end{aligned}
$$

where $R(K)=\int K^{2}(u) \mathrm{d} u$ and $k_{2}(K)=\int u^{2} K(u) \mathrm{d} u$ denote constants depending on the kernel function. This result demonstrates the well-known problem in selecting an appropriate bandwidth, often termed the "variancebias trade-off". For the bias to decrease one needs to select a small bandwidth; however, taking this parameter small means automatically an increase in the variance. The variance-bias trade-off is in accordance with the intuitive understanding of smoothing as a technique to reveal the underlying structure of the data by reducing the "noise" (variance) at the expense of some "oversimplification" (bias). More details on these general aspects of smoothing and on the technical details of the derivation of asymptotic results in a general counting process framework can be found in the monograph by Andersen, Borgan, Gill and Keiding (1993).

\section{Large-sample properties of visual error criteria}

The standard asymptotic results on the properties of the kernel estimator $\widehat{h}$ consider only distances between $\widehat{h}(x)$ and $h(x)$ at fixed $x \in \mathcal{R}_{+}$and thus exhibit the drawback of measuring error only vertically as discussed previously. In this section we analyse the asymptotic behaviour of the quadratic versions of the visual error criteria that is essentially determined by the asymptotics of the distances $d\left((x, \widehat{h}(x)), G_{h}\right)$ and $d\left((x, h(x)), G_{\widehat{h}}\right)$, respectively, for $n \rightarrow \infty$. To this end, consider for fixed $x_{0} \in \mathcal{R}_{+}$the distances as sequences of nonnegative random variables $D_{n}^{1}\left(x_{0}\right)$ and $D_{n}^{2}\left(x_{0}\right)$, respectively. 
In the context of density estimation Marron and Tsybakov (1995) derived a result on the convergence in probability of $D_{n}^{1}\left(x_{0}\right)$ and $D_{n}^{2}\left(x_{0}\right)$, respectively, for $n \rightarrow \infty$. In the proof they used a combination of primarily geometric arguments that can be directly transferred to the hazard rate context. The only "density-specific" step concerned the convergence in probability of the kernel estimator for the derivative of the density to the true derivative of the density. The same property, i.e. $\widehat{h}^{\prime}\left(x_{0}\right) \rightarrow_{P} h^{\prime}\left(x_{0}\right)$ for fixed $x_{0}$ as $n \rightarrow \infty$, holds for the kernel estimator of hazard rate, modulo a sufficiently correct choice of the bandwidth, as can be checked easily. Thus, analogous to the density context analysed in Marron and Tsybakov (1995),

$$
n^{2 / 5}\left(D_{n}^{i}\left(x_{0}\right)-\frac{\left|\widehat{h}\left(x_{0}\right)-h\left(x_{0}\right)\right|}{\sqrt{1+h^{\prime}\left(x_{0}\right)^{2}}}\right) \stackrel{\mathcal{P}}{\rightarrow} 0
$$

holds in the hazard rate context for both $i=1$ and $i=2$ as $n \rightarrow \infty$. This result allows us to build an asymptotic bridge between the expected squared visual error criteria and the conventional approach as follows:

$$
\begin{aligned}
\mathrm{E}\left[\mathrm{VE}_{2}(\widehat{h} \rightarrow h)^{2}\right] & =\mathrm{E} \int d\left((x, \widehat{h}(x)), G_{h}\right)^{2} \mathrm{~d} x \\
& =\int \mathrm{E}\left(D_{n}^{1}(x)^{2}\right) \mathrm{d} x \\
& \approx \int \frac{\mathrm{E}\left([\widehat{h}(x)-h(x)]^{2}\right)}{1+h^{\prime}(x)^{2}} \mathrm{~d} x \\
& \approx \int \frac{\mu^{2}(x)+\sigma^{2}(x)}{1+h^{\prime}(x)^{2}} \mathrm{~d} x .
\end{aligned}
$$

Here the first two equalities are given by the definition of the quantities, the first approximation utilises the asymptotic result on $D_{n}^{1}$, and the last approximation results from plugging in the asymptotic MSE expression given in the previous section (a similar line of reasoning leads to the same asymptotic result for $\left.\mathrm{E}\left[\mathrm{VE}_{2}(h \rightarrow \widehat{h})^{2}\right]\right)$. When defining $w(x)=\left(1+h^{\prime}(x)^{2}\right)^{-1}$, the final expression above can also be viewed as the standard asymptotic representation of a weighted MISE employing the special weight function $w(\cdot)$. This shows that expected squared visual error corresponds asymptotically to a weighted MISE, and inspection of the weight function allows one to infer in which situations the two concepts of measuring discrepancy between curves will contradict each other. For example, for linear functions both error concepts give identical answers, but for functions with regions where $\left|h^{\prime}(x)\right|$ is large $\mathrm{E}\left[\mathrm{VE}_{2}(\cdot)^{2}\right]$ and MISE can disagree markedly. This finding corresponds with the motivating example given in Section 2 and the intuitive understanding of the deficiencies of the $L_{2}$-approach in situations where functions reveal several peaks. 
Although the asymptotic expressions for

$$
\mathrm{E}\left[\mathrm{VE}_{2}(\widehat{h} \rightarrow h)^{2}\right] \text { and } \mathrm{E}\left[\mathrm{VE}_{2}(h \rightarrow \widehat{h})^{2}\right]
$$

are the same, details of the proof in Marron and Tsybakov (1995) suggest that the asymptotics might come into action later for $\mathrm{VE}_{2}(h \rightarrow \widehat{h})$ than for $\mathrm{VE}_{2}(\hat{h} \rightarrow h)$. This should also be reflected in a different finite sample behaviour of the two criteria which needs further investigation.

From the findings on the two asymmetrical versions of squared visual error it follows immediately for the symmetrised criterion $\mathrm{SE}_{2}(\widehat{h}, h)$ that when $n$ is large,

$$
\mathrm{E}\left[\mathrm{SE}_{2}(\widehat{h}, h)^{2}\right]=\mathrm{E}\left[\mathrm{VE}_{2}(h \rightarrow \widehat{h})^{2}+\mathrm{VE}_{2}(\widehat{h} \rightarrow h)^{2}\right] \approx 2 \int \frac{\mu^{2}(x)+\sigma^{2}(x)}{1+h^{\prime}(x)^{2}} \mathrm{~d} x .
$$

Remark 1. Implicit in our definition of $\mathrm{VE}_{2}(h \rightarrow \widehat{h})$ and $\mathrm{VE}_{2}(\widehat{h} \rightarrow h)$ in Section 2 , and hence of the symmetrised criterion $\mathrm{SE}_{2}(\widehat{h}, h)$, is the absence of any $x$-weight function that otherwise could have been utilised to mark relative importance of certain $x$-regions. Sometimes such $x$-weighting could be natural, however. Our definitions and the large-sample results above generalise immediately to say

$$
\left[\int d\left((x, h(x)), G_{\widehat{h}}\right)^{2} v(x) \mathrm{d} x\right]^{1 / 2} \text { and } \quad\left[\int d\left((x, \widehat{h}(x)), G_{h}\right)^{2} v(x) \mathrm{d} x\right]^{1 / 2}
$$

and their consequent symmetrised version $\mathrm{SE}_{2}(\cdot ; v)$, say, for any chosen $v(x)$. The large-sample result relating this to another weighted MISE measure is that

$$
\mathrm{E}\left[\mathrm{SE}_{2}(\widehat{h}, h ; v)^{2}\right] \approx 2 \int \frac{\mu^{2}(x)+\sigma^{2}(x)}{1+h^{\prime}(x)^{2}} v(x) \mathrm{d} x .
$$

One might for example use $v$ equal or close to 1 over the time interval deemed most important and equal or close to 0 over the rest. There are also reasons of technical necessity to allow $v$ functions to be present; in order for the result above to hold over the full time interval $[0, \infty)$ one would need certain convergence in probability statements, also including the derivative estimate, to hold uniformly in $x$. This could lead to technical conditions that might be hard to secure on the full halfline, but the result above would nevertheless hold for classes of $v(x)$ functions that tend suitably to zero as $x$ grows.

Remark 2. It is also worth pointing out in this connection that the traditional MISE criterion as such also could and sometimes should be used with a weight function $v(x)$. Hazard rate estimators often err much more for large time values, for which there are simply few individuals left at risk, than 
for smaller time values. Hence the unweighted $\int(\widehat{h}-h)^{2} \mathrm{~d} x$ could easily be dominated by what happens for large $x$ only. The remedy is again to use a weight function $v(x)$ inside the integral, perhaps one going smoothly towards zero. A case in point is the approximate relation to the MISE of density estimation. The density $f(x)$ and its estimate $\widehat{f}(x)$ can be represented as $h(x) \exp [-H(x)]$ and $\widehat{h}(x) \exp [-\widehat{H}(x)]$ respectively, where $H$ and $\widehat{H}$ are the cumulative sisters of $h$ and $\widehat{h}$. The unweighted ISE and MISE criteria have long standings in the density estimation sphere, which is to say that

$$
\int(\widehat{f}-f)^{2} \mathrm{~d} x=\int[\widehat{h} \exp (-\widehat{H})-h \exp (-H)]^{2} \mathrm{~d} x
$$

is a reasonable criterion. But this is approximately equal to $\int \exp (-2 H)$ $(\widehat{h}-h)^{2} \mathrm{~d} x$. In other words, claiming appropriateness of the MISE measure in density estimation leads one to accept appropriateness of the weighted MISE criterion for hazard rate estimation, with weight function of the type $\exp (-2 H)=(1-F)^{2}$.

Remark 3. We have discussed visual error criteria and a large-sample connection to a weighed MISE criterion in terms of the traditional kernel estimator for hazard rates (see Section 4). However, results very similar to those above can be reached for a host of recently developed competing hazard rate estimators; see Hjort (1996) for a generous list. Such a competitor would have a different $\mu(x)$ and possibly a different $\sigma(x)$, but the structure of the large-sample result above, and hence its main consequences, would be the same.

\section{Discussion}

The standard approach when evaluating the performance of a functional estimate uses some $L$-norm to quantify its distance to the true function. As demonstrated in this paper, there are several situations in which this approach contradicts the graphical notion of discrepancy between curves since all $L$-norms consider only vertical distances and neglect aspects of qualitative similarities. Thus, the new concept of visual error criteria has been discussed as an alternative method to evaluate the visual appropriateness of functional estimates. In the context of hazard rate estimation from censored data it has been shown that application of these criteria corresponds asymptotically to a weighted version of the conventional MISE. A more detailed analysis of the finite sample properties of visual error criteria is, however, needed to expand the knowledge about advantages and disadvantages of this new concept. Visual error criteria represent an attractive first step into the direction of rethinking the mathematical evaluation of the performance of functional estimates, but they require further elaboration - and perhaps some modification - prior to their routine application. 


\section{References}

ANDERSEN, P.K., BORGAN, Ø., GILL, R.D. and KEIDING, N. (1993): Statistical Models Based on Counting Processes. Springer-Verlag, New York.

GEFELLER, O. and DETTE, H. (1992): Nearest neighbour kernel estimation of the hazard function from censored data. Journal of Statistical Computation and Simulation 43, 93-101.

GEFELLER, O. and MICHELS, P. (1992): Nichtparametrische Analyse von Verweildauern. Österreichische Zeitschrift für Statistik und Informatik 22, 37-59.

HÄRDLE, W. (1991): Applied Nonparametric Regression. Cambridge University Press, Boston.

HJORT, N.L. (1996): New methods for hazard rate estimation. Invited lecture at the XVII ${ }^{\mathrm{eme}}$ Rencontre Franco-Belge de Staticiens, Université de Marne-la-Vallée, 1996; summary published in the proceedings of that conference, p. 20-24.

KOOPERBERG, C. and STONE, C.J. (1991): A study of logspline density estimation. Computational Statistics \& Data Analysis 12, 327-347.

MARRON, J.S. and TSYBAKOV, A.B. (1995): Visual error criteria for qualitative smoothing. Journal of the American Statistical Association 90, 499-507.

MÜLLER, H.G. and WANG, J.L. (1994): Hazard rate estimation under random censoring with varying kernels and bandwidths. Biometrics 50, 61-76.

PARZEN, E. (1962): On the estimation of a probability density and mode. Annals of Mathematical Statistics 33, 1065-1076.

ROSENBLATT, M. (1956): Remarks on some nonparametric estimates of a density function. Annals of Mathematical Statistics 27, 832-837.

SCHÄFER, H. (1985): A note on data-adaptive kernel estimation of the hazard and density function in the random censorship situation. Annals of Statistics 13, 818-820.

WAND, M.P. and JONES, M.C. (1995): Kernel Smoothing. Chapman and Hall, London. 